\title{
Density functional theory calculations and Hirshfeld surface analysis of propyl-para-hydroxybenzoate (PHB) for optoelectronic application
}

\author{
V. Mohankumar ${ }^{1, *}$, N. Karunagaran ${ }^{2}$, M. Senthil Pandian ${ }^{1}$, P. RAmasamy ${ }^{1}$ \\ ${ }^{1}$ SSN Research Centre, SSN College of Engineering, Chennai - 603 110, Tamil Nadu, India \\ 2Department of Physics, SRM Institute of Science and Technology, Ramapuram Campus, Bharathi Salai, Ramapuram, Chennai, TN, India
}

\begin{abstract}
The geometries, electrostatic potential, Mulliken charge analysis, Natural Bond Orbital analysis and polarizabilities of propyl-para-hydroxybenzoate were calculated using B3LYP functional with 6-311++G(d,p) basis set. The calculated geometries are well matched with the experimental values. The Mullliken atomic charge analysis shows that the eventual charges are contained in the molecule. The NBO analysis explains the intramolecular charge transfer in the PHB molecule. The bonding features of the molecule were analyzed with the aid of Hirshfeld surface analysis. The frontier molecular orbital analysis showed the charge transfer obtained within the molecule. The calculated hyperpolarizability of the PHB molecule was $6.977 \mathrm{E}^{-30}$ esu and it was 8.9 times that of standard urea molecule.
\end{abstract}

Keywords: Density Functional Theory; HOMO-LUMO; NLO; Hirshfeld surface

\section{Introduction}

Nonlinear optics (NLO) deals with the conversion of applied electromagnetic field in various materials into new electromagnetic field altered in phase, frequency, amplitude or other physical properties $[1,2]$. The architecture of nonlinear optical molecules has become a spotlight of modern research community. In their vision there are the potential applications in different photonic technologies. Organic nonlinear optical (NLO) materials have gained special consideration in recent years due to their second or third-order hyperpolarizabilities compared to inorganic NLO materials and their applications in optical devices, such as optical switches, optical modulators, optical communications and optical data storage [3-5]. Organic compounds, compared to the more traditional inorganic compounds, induce electro-optic effects and produce large nonlinear optical response due to the large delocalization of $\pi$ electrons. For the production of organic nonlinear optical (NLO)

*E-mail: mohaphysics@gmail.com compounds, donor and/or acceptor substituents such as azo-, amino-, nitro-, cyano-, halo-, hydroxyls are used. Azo compounds that are one of the important constituents of synthetic organic dyes contain nitrogen as the azo $(-\mathrm{N}=\mathrm{N}-)$ group. They are used as colorants in the textile, paper, cosmetics, drugs, food coloring, and other consumer goods. They are promising systems for dyestuffs, $\mathrm{pH}$ indicators and optical applications such as holographic and digital storage, polarizing films, lasers, liquid crystal command surfaces and nonlinear optical devices. In recent years, computational chemistry has become an important tool for chemists and a wellaccepted instrument in experimental chemistry [611]. In design of new molecules for optoelectronic applications, quantum chemical calculations are attractive method and they can also be used to find some of molecular properties, such as molecular dipole moment and polarizability [12, 13]. Density functional theory calculations offer invaluable add for the experimental results. Using vibrational spectroscopy, a lot of studies have been carried out over last years on polyconjugated arrangements in order to study their structural properties. 
Infrared and Raman spectroscopy have facilitated us to gather information on the molecular structure of conjugated molecular systems and their NLO nature [14]. The crystal structure was reported by Zhou et al. [15]. Growth and characterization of propyl-para-hydroxybenzoate single crystals were investigated by Karunagaran et al. [16] and Solanki et al. [17].

To the best of authors knowledge there is no theoretical study on the propyl-para-hydroxybenzoate. In the present study, the structure, HOMO-LUMO energy gap, Mulliken population analysis, natural bond orbital (NBO) analysis, hyperpolarizabilities and Hirshfeld surface of the organic nonlinear optical PHB molecule are investigated. The NBO analysis explains the consequent orbital interactions within the molecule.

\section{Materials and methods}

The title compound PHB is commercially available and has been used for growth. PHB single crystals have been grown using slow evaporation solution growth technique at room temperature. The saturated solution of PHB was obtained by dissolving the material in methanol solvent with continuous stirring of the solution at room temperature and this solution was filtered using 41 grade Whatman filter paper to remove the suspended impurities. The beaker containing the filtered solution was properly closed for controlled evaporation. We also could control the volatilization rate of solvent through drilling a certain number of fine pores on the air-tight cover [16].

\section{Results and discussions}

\subsection{Optimized molecular structure}

The optimized molecular structure of PHB with atom numbering scheme is presented in Fig. 1. The optimized molecular structural parameters of PHB are listed in Table 1. The XRD values are compared with corresponding optimized parameters and minor variations are found between them [16]. The optimized parameters are slightly larger than the experimental values and this may be due to the fact that the theoretical calculations have been performed on an isolated molecule in the gas phase. The $\mathrm{C}-\mathrm{H}$ bond lengths are in the range of $1.082 \AA$ to $1.095 \AA$ in DFT and the range between $0.93 \AA$ and $0.971 \AA$ are obtained from experimental data. $\mathrm{C}-\mathrm{O}$ bond lengths are in the range of $1.212 \AA$ to $1.450 \AA$ in DFT and the range between $1.208 \AA$ and $1.450 \AA$ is obtained in XRD. The $\mathrm{C}-\mathrm{O}$ bond lengths are nearly matched with experimental values, the C11-O23 bond length is slightly lower than the other $\mathrm{C}-\mathrm{O}$ bond lengths due to the double bond between carbon and oxygen atoms. The root mean square error (RMSE) is found to be $0.097 \AA$ and $1.848^{\circ}$ for bond lengths and bond angles, respectively. The correlation coefficients are depicted in Fig. 2. The correlation coefficients of bond lengths and bond angles are 0.9489 and 0.8948 , respectively. From the RMSE values and correlation coefficients, it is concluded that the geometrical parameters are well matched with each other.

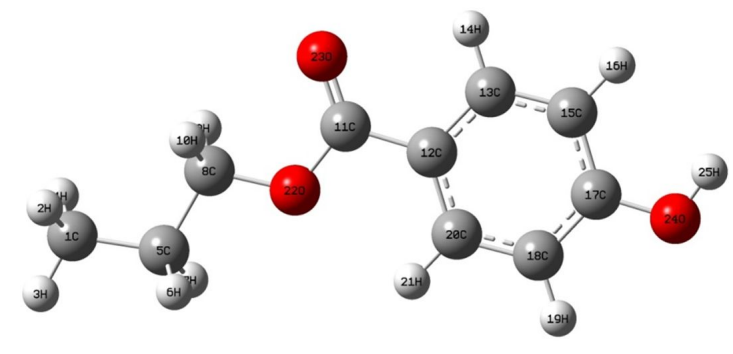

Fig. 1. Optimized molecular structure of PHB.
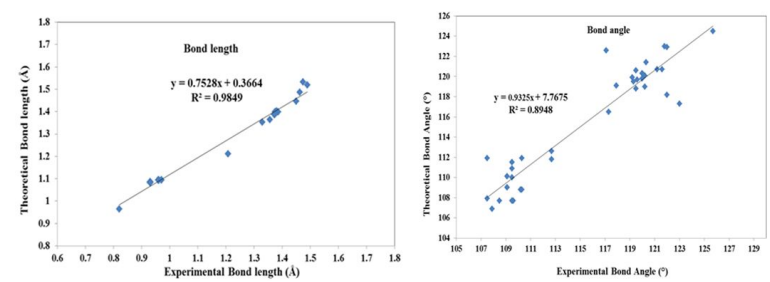

Fig. 2. Correlation coefficients of bond lengths and bond angles.

\subsection{Natural bond orbital analysis (NBO)}

The natural bond orbital analysis is a powerful tool for analyzing the chemical processes and hyperconjugative interactions in the molecule $[18$, 19]. The NBO of the PHB molecule was analyzed 
Table 1. Optimized geometrical parameters of PHB.

\begin{tabular}{|c|c|c|}
\hline \multicolumn{3}{|c|}{ Bond length $[\AA]$} \\
\hline Parameters & DFT & XRD \\
\hline$(\mathrm{C} 1-\mathrm{H} 2)$ & 1.095 & 0.959 \\
\hline (C1-H3) & 1.093 & 0.96 \\
\hline (C1-H4) & 1.095 & 0.961 \\
\hline (C1-C5) & 1.532 & 1.474 \\
\hline (C5-H6) & 1.095 & 0.97 \\
\hline (C5-H7) & 1.095 & 0.97 \\
\hline (C5-C8) & 1.519 & 1.49 \\
\hline (C8-H9) & 1.094 & 0.971 \\
\hline (C8-H10) & 1.094 & 0.971 \\
\hline (C8-O22) & 1.447 & 1.45 \\
\hline (C11-C12) & 1.486 & 1.463 \\
\hline (C11-O22) & 1.352 & 1.329 \\
\hline (C11-O23) & 1.212 & 1.208 \\
\hline (C12-C13) & 1.399 & 1.386 \\
\hline (C12-C20) & 1.403 & 1.381 \\
\hline (C13-H14) & 1.083 & 0.931 \\
\hline (C13-C15) & 1.388 & 1.372 \\
\hline (C15-H16) & 1.086 & 0.931 \\
\hline (C15-C17) & 1.399 & 1.377 \\
\hline (C17-C18) & 1.398 & 1.374 \\
\hline (C17-O24) & 1.364 & 1.357 \\
\hline (C18-H19) & 1.084 & 0.93 \\
\hline (C18-C20) & 1.387 & 1.375 \\
\hline (C20-H21) & 1.082 & 0.931 \\
\hline (O24-H25) & 0.964 & 0.82 \\
\hline \multicolumn{3}{|c|}{ Bond angle $\left[{ }^{\circ}\right]$} \\
\hline Parameters & DFT & XRD \\
\hline$(\mathrm{H} 2-\mathrm{C} 1-\mathrm{H} 3)$ & 107.7 & 109.6 \\
\hline$(\mathrm{H} 2-\mathrm{C} 1-\mathrm{H} 4)$ & 107.7 & 109.5 \\
\hline (H2-C1-C5) & 111.5 & 109.5 \\
\hline$(\mathrm{H} 3-\mathrm{C} 1-\mathrm{H} 4)$ & 107.7 & 109.5 \\
\hline (H3-C1-C5) & 110.9 & 109.5 \\
\hline (H4-C1-C5) & 111.5 & 109.5 \\
\hline (C1-C5-H6) & 110.1 & 109.1 \\
\hline$(\mathrm{C} 1-\mathrm{C} 5-\mathrm{H} 7)$ & 110.1 & 109.1 \\
\hline (C1-C5-C8) & 111.8 & 112.7 \\
\hline (H6-C5-H7) & 106.9 & 107.9 \\
\hline (H6-C5-C8) & 109 & 109.1 \\
\hline (H7-C5-C8) & 109 & 109.1 \\
\hline (C5-C8-H9) & 111.9 & 110.3 \\
\hline (C5-C8-H10) & 111.9 & 107.5 \\
\hline (C5-C8-O22) & 107.9 & 107.5 \\
\hline (H9-C8-H10) & 107.7 & 108.5 \\
\hline (H9-C8-O22) & 108.8 & 110.2 \\
\hline (H10-C8-O22) & 108.8 & 110.3 \\
\hline (C12-C11-O22) & 112.6 & 112.7 \\
\hline (C12-C11-O23) & 124.5 & 125.7 \\
\hline (O22-C11-O23) & 123 & 121.8 \\
\hline (C11-C12-C13) & 118.2 & 122 \\
\hline (C11-C12-C20) & 122.9 & 122 \\
\hline (C13-C12-C20) & 119.1 & 117.9 \\
\hline (C12-C13-H14) & 118.8 & 119.5 \\
\hline (C12-C13-C15) & 120.7 & 121.2 \\
\hline (H14-C13-C15) & 120.6 & 119.5 \\
\hline (C13-C15-H16) & 120.3 & 120 \\
\hline (C13-C15-C17) & 119.8 & 120 \\
\hline (H16-C15-C17) & 120.1 & 120.2 \\
\hline (C15-C17-C18) & 120.2 & 120.1 \\
\hline (C15-C17-O24) & 122.6 & 117.1 \\
\hline (C18-C17-O24) & 117.3 & 123 \\
\hline (C17-C18-H19) & 119 & 120.2 \\
\hline (C17-C18-C20) & 119.7 & 119.6 \\
\hline (H19-C18-C20) & 121.4 & 120.3 \\
\hline (C12-C20C18) & 120.7 & 121.6 \\
\hline (C12-C20-H21) & 119.5 & 119.3 \\
\hline (C18-C20-H21) & 119.9 & 119.2 \\
\hline
\end{tabular}

using B3LYP/6-311++G(d,p) level. The NBO has been listed in Table 2. The NBO table shows the intramolecular charge transfer within the molecule. The strong intramolecular charge transfer interaction raises the interaction energy and stabilizes the molecular system. The hyperconjugative energy was obtained from the following equation:

$$
E_{2}=\Delta E_{i j}=q_{i} \frac{F(i, j)^{2}}{\left(\varepsilon_{j}-\varepsilon_{i}\right)}
$$

where $q_{i}$ is the donor orbital occupancy, $E_{i}, E_{j}$, are the diagonal elements and $F_{i j}$ is the offdiagonal NBO Fock matrix element. From the table, lone pair electrons of $\mathrm{LP}(\mathrm{O} 22)$ to antibonding orbital $\pi^{*}(\mathrm{C} 11-\mathrm{O} 23)$ have high stabilization energy of $45.19 \mathrm{kcal} \mathrm{mol}^{-1}$. The lone pair electrons of $\mathrm{LP}(\mathrm{O} 22)$ to antibonding orbital $\pi^{*}(\mathrm{C} 11-\mathrm{O} 22)$ have the next highest stabilization energy of $32.31 \mathrm{kcal} \mathrm{mol}^{-1}$. The title molecule has been stabilized and this has been confirmed by NBO analysis.

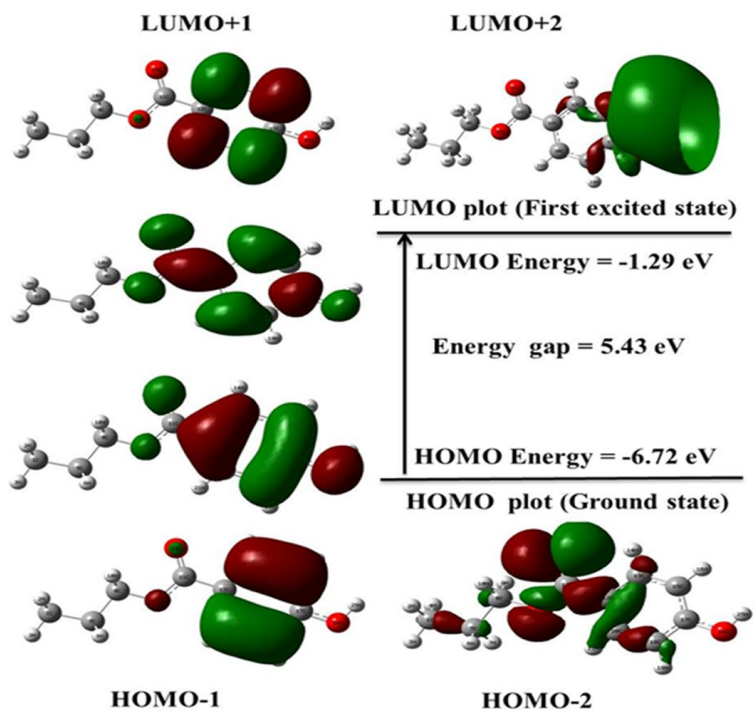

Fig. 3. Frontier molecular orbitals of PHB.

\subsection{Frontier molecular orbital}

The chemical reactivity and kinetic stability of the molecule were characterized with the aid of frontier molecular orbital gap. A molecule with lesser frontier orbital gap is highly polarizable and 
Table 2. Second order Fock matrix in NBO basis for PHB molecule, E (2) denotes energy of hyperconjugative interactions. $E(j)-E(i)$ denotes the energy difference between donor and acceptor $i$ and $j$ natural bond charges (NBO) orbitals. F (i,j) is the Fock matrix element between $i$ and $j \mathrm{NBO}$ orbitals.

\begin{tabular}{ccccccccc}
\hline Donor (i) & Type & ED(i) (e) & Acceptor (j) & Type & ED(j) (e) & $\begin{array}{c}\mathrm{E}(2) \\
{\left[\mathrm{kcal} \cdot \mathrm{mol}^{-1}\right]}\end{array}$ & $\begin{array}{c}\mathrm{E}(\mathrm{j})-\mathrm{E}(\mathrm{i}) \\
{[\mathrm{a} . \mathrm{u} .]}\end{array}$ & $\begin{array}{c}\mathrm{F}(\mathrm{i}, \mathrm{j}) \\
{[\mathrm{a} . \mathrm{u} .]}\end{array}$ \\
\hline \hline $\mathrm{O} 22$ & $\mathrm{LP}$ & 1.798 & $\mathrm{C} 11-\mathrm{O} 23$ & $\pi^{*}$ & 0.264 & 45.19 & 0.34 & 0.112 \\
$\mathrm{O} 23$ & LP & 1.850 & $\mathrm{C} 11-\mathrm{O} 22$ & $\sigma^{*}$ & 0.098 & 32.31 & 0.63 & 0.129 \\
& & & $\mathrm{C} 11-\mathrm{C} 12$ & $\sigma^{*}$ & 0.063 & 17.01 & 0.69 & 0.099 \\
$\mathrm{O} 24$ & LP & 1.868 & $\mathrm{C} 15-\mathrm{C} 17$ & $\pi^{*}$ & 0.390 & 28.71 & 0.35 & 0.096 \\
$\mathrm{C} 15-\mathrm{C} 17$ & $\pi$ & 1.635 & $\mathrm{C} 12-\mathrm{C} 13$ & $\pi^{*}$ & 0.380 & 25.06 & 0.3 & 0.077 \\
$\mathrm{C} 18-\mathrm{C} 20$ & $\pi$ & 1.692 & $\mathrm{C} 15-\mathrm{C} 17$ & $\pi^{*}$ & 0.390 & 24.13 & 0.27 & 0.074 \\
$\mathrm{C} 18-\mathrm{C} 20$ & $\pi$ & 1.692 & $\mathrm{C} 12-\mathrm{C} 13$ & $\pi^{*}$ & 0.380 & 16.06 & 0.29 & 0.062 \\
$\mathrm{C} 12-\mathrm{C} 13$ & $\pi$ & 1.640 & $\mathrm{C} 18-\mathrm{C} 20$ & $\pi^{*}$ & 0.300 & 23.17 & 0.28 & 0.073 \\
& & & $\mathrm{C} 11-\mathrm{O} 23$ & $\pi^{*}$ & 0.264 & 22.21 & 0.27 & 0.071 \\
$\mathrm{C} 15-\mathrm{C} 17$ & $\pi$ & 1.635 & $\mathrm{C} 18-\mathrm{C} 20$ & $\pi^{*}$ & 0.300 & 14.76 & 0.3 & 0.06 \\
$\mathrm{O} 24$ & $\mathrm{LP}$ & 1.979 & $\mathrm{C} 15-\mathrm{C} 17$ & $\sigma^{*}$ & 0.027 & 6.00 & 1.17 & 0.075 \\
$\mathrm{O} 22$ & $\mathrm{LP}$ & 1.964 & $\mathrm{C} 11-\mathrm{O} 23$ & $\sigma^{*}$ & 0.018 & 7.17 & 1.17 & 0.082 \\
\hline
\end{tabular}

Table 3. HOMO, LUMO, Energy gap and global indices values.

\begin{tabular}{ll}
\hline HOMO & $-6.72 \mathrm{eV}$ \\
LUMO & $-1.29 \mathrm{eV}$ \\
Energy gap & $5.43 \mathrm{eV}$ \\
Electronegativity $(\chi)$ & $2.715 \mathrm{eV}$ \\
Chemical hardness $(\mathrm{\eta})$ & $4.005 \mathrm{eV}$ \\
Chemical softness $(\mathrm{S})$ & $0.184 \mathrm{eV}^{-1}$ \\
\hline
\end{tabular}

has high chemical reactivity, low kinetic stability and it is also named as a soft molecule [20]. The HOMO is donating the electron, while LUMO is electron acceptor. The FMOs of PHB are depicted in Fig. 3. From the pictorial of FMOs, the HOMO-2 is mainly localized on the bridge of the molecule. HOMO-1 is localized on the phenol ring of PHB molecule. HOMO is localized on phenol part and bridge of the molecule. LUMO is mainly localized on the phenol ring and the $\mathrm{C}=\mathrm{O}$ group of the molecule. The HOMO and LUMO electron densities are located in same part of the molecule. HOMO and LUMO show $\pi$ and $\sigma$ bond characteristics respectively. LUMO+1 is delocalized on phenol part of the molecule. The LUMO+2 of the molecule is delocalized on the $\mathrm{OH}$ part of the molecule. From the FMO analysis, it has been concluded that the charges are transferred within the molecule. The calculated HOMO-LUMO energy gap is $5.43 \mathrm{eV}$. By using HOMO-LUMO energy values, global indices such as electronegativity, chemical hardness and chemical softness of the molecule can be calculated. $\eta=(I-A) / 2$ (chemical hardness), $\chi=(\mathrm{I}+\mathrm{A}) / 2$ (electronegativity) and $S=1 / 2 \eta$ (chemical softness), where I and $\mathrm{A}$ are ionization potential and electron affinity; $\mathrm{I}=-\mathrm{E}_{\mathrm{HOMO}}$ and $\mathrm{A}=-\mathrm{E}_{\mathrm{LUMO}}[21,22]$. The calculated parameters are listed in Table 3.

\subsection{Molecular electrostatic potential (MEP)}

To understand the hydrogen bonding interactions [23] and the electrophilic and nucleophilic sites [24], the charge distribution gives useful information. 3D pictorial of molecular electrostatic potential (MEP) map of PHB is presented in Fig. 4. From the MEP figure, the negative charge is delocalized on oxygen atoms and identified by yellowish color. These negative charges on the oxygen atoms are expected and the electrostatic potential map corresponds to electronegativity and partial charge.

The carbon ring and hydrogen atoms are pointed out by greenish color. It shows that 


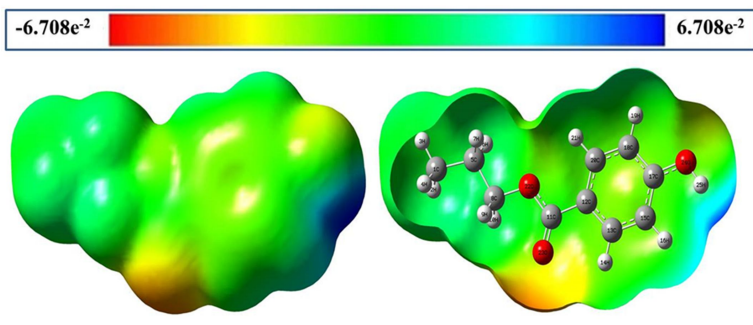

Fig. 4. Molecular electrostatic potential map of PHB.

the positive potential is delocalized over the carbon and hydrogen atoms. The range of the color code is between -6.708 electrons per cubic Bohr to 6.708 electrons per cubic Bohr. The blue color demonstrates the strong repulsion and red color demonstrates the strong attraction. The MEP analysis gives a clue where the intermolecular interactions are formed within the molecule.

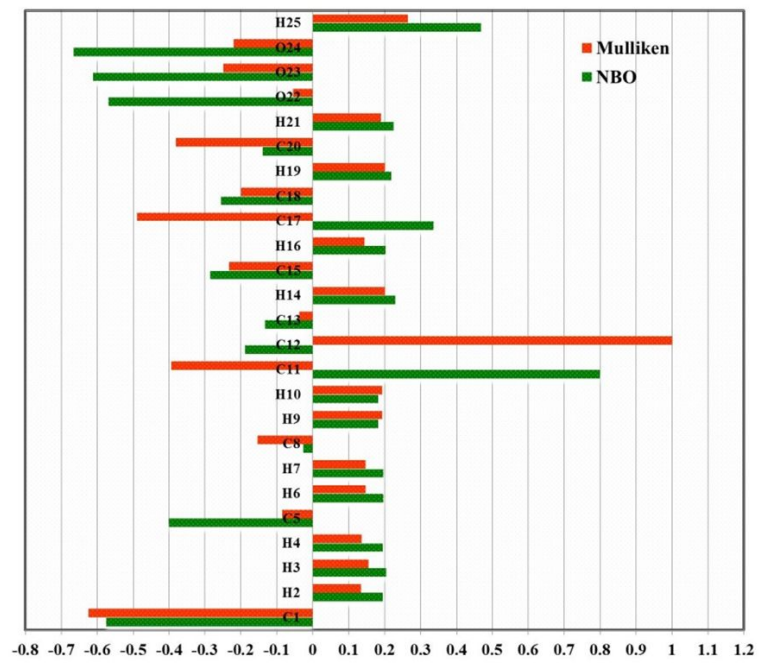

Fig. 5. Natural and Mulliken charges of PHB.

\subsection{Mulliken charge analysis}

The natural charge and the Mulliken atomic charge of PHB molecule was calculated by B3LYP/6-311++G(d,p) basis set. The pictorial representation of charge disposal in the molecule is depicted in Fig. 5. All the carbon atoms are negatively charged except $\mathrm{C} 12$. The $\mathrm{C} 12$ has positive value of $(1.002 \mathrm{e})$. It is due to the electronegativity of the atom present in the next carbon atom. All the hydrogen atoms have positive charge and $\mathrm{H} 25$ has the highest Mulliken charge compared to the other hydrogen atoms. It is attached to the electronegative atom $\mathrm{O} 24$. This charge analysis gives us the clue for charge transfer within the title molecule.

\subsection{Polaraizability and hyperpolaraiz- ability}

The NLO response of the isolated molecule in an electric field $\mathrm{E}_{\mathrm{i}}(\omega)$ can be presented in Taylor series expansion of the total dipole moment $\mu_{\text {tot }}$, induced by the field and it is given by following expression:

$$
\mu_{t o t}=\mu_{0}+\alpha_{i j} E_{j}+\beta_{i j k} E_{j} E_{k}+\ldots
$$

where $\alpha$ is the linear polarizability, $\mu_{0}$ is the permanent dipole moment and $\beta_{\mathrm{ijk}}$ are the hyperpolarizability tensor components. The average linear polarizability is defined as [25]:

$$
\alpha_{t o t}=\left(\alpha_{x x}+\alpha_{y y}+\alpha_{z z}\right) / 3
$$

First hyperpolarizability is a third rank tensor and this can be described by $3 \times 3 \times 3$ matrix. The 27 components are reduced to 10 components due to Kleinman symmetry [26].

Table 4. Hyperpolarizability components.

\begin{tabular}{cc}
\hline$\beta$ components & $\beta$ [a.u.] \\
\hline \hline$\beta_{\mathrm{xxx}}$ & -739.414 \\
$\beta_{\mathrm{xxy}}$ & 324.325 \\
$\beta_{\mathrm{xyy}}$ & 1.353 \\
$\beta_{\mathrm{yyy}}$ & -53.432 \\
$\beta_{\mathrm{xxz}}$ & 0.051 \\
$\beta_{\mathrm{xyz}}$ & -0.016 \\
$\beta_{\mathrm{yyz}}$ & 0.003 \\
$\beta_{\mathrm{xzz}}$ & -18.234 \\
$\beta_{\mathrm{yzz}}$ & 12.104 \\
$\beta_{\mathrm{zzz}}$ & 0.007 \\
$\beta_{\mathrm{tot}}$ & $6.97 \mathrm{E}^{-30} \mathrm{esu}$ \\
\hline
\end{tabular}

The output from Gaussian 09 provides 10 components of this matrix as $\beta_{\mathrm{xxx}}, \beta_{\mathrm{xxy}}, \beta_{\mathrm{xyy}}, \beta_{\mathrm{yyy}}, \beta_{\mathrm{xxz}}, \beta_{\mathrm{xyz}}, \beta_{\mathrm{yyz}}, \beta_{\mathrm{xzz}}, \quad \beta_{\mathrm{yzz}}$, $\beta_{\text {zzz }}$, respectively. The hyperpolaraizability components are listed in Table 4. Using the $\mathrm{x}, \mathrm{y}$ 
and $\mathrm{z}$ components the magnitude of the first hyperpolaraizability tensor $\beta$ can be calculated by:

$$
\beta_{t o t}=\left(\beta_{x}^{2}+\beta_{y}^{2}+\beta_{z}^{2}\right)^{1 / 2}
$$

The complete equation for calculating $\beta$ is given by the following equation:

$$
\begin{aligned}
& \beta_{t o t}=\left(\left(\beta_{x x x}^{2}+\beta_{x y y}^{2}+\beta_{x z z}^{2}\right)+\left(\beta_{y y y}^{2}+\beta_{y z z}^{2}+\beta_{y x x}^{2}\right)\right. \\
& \left.+\left(\beta_{z z z}^{2}+\beta_{z x x}^{2}+\beta_{z y y}^{2}\right)\right)^{1 / 2}
\end{aligned}
$$

The electronic dipole moment, polaraizability and hyperpolaraizability of the title compound were calculated at the B3LYP/6-311++G(d,p) level using gaussian $09 \mathrm{~W}$ package. The calculated values of $\alpha_{\text {tot }}$ and $\beta_{\text {tot }}$ for the title compound are $1.96 \mathrm{E}^{-23}$ esu and $6.977 \mathrm{E}^{-30} \mathrm{esu}$, respectively. The $\beta_{\text {tot }}$ of the title molecule is 8.9 times greater than that of the standard urea molecule and $\beta_{\text {tot }}$ values are calculated in same level of theory. The calculated values are in atomic units and the calculated values are converted into electrostatic units (esu) ( 1 a.u. $=0.1482 \times 10^{-24}$ esu for $\alpha$ and 1 a.u. $=$ $\left.8.6393 \times 10^{-33} \mathrm{esu}\right)$.

\subsection{Hirshfeld surface}

The intermolecular interactions in a crystal structure have been analyzed by Hirshfeld surface analysis (HS). The fingerprint plot of PHB demonstrates the clue for intermolecular interaction patterns. The Hirshfeld surfaces of PHB are depicted in Fig. 6. Two distances have been measured in the Hirshfeld plot. The two distances are $d_{e}$ and $d_{i}$, where $d_{e}$ represents the distance from the point to the nearest nucleus external to the surface and $d_{i}$ represents the distance to the nearest nucleus internal to the surface. These two distances $d_{e}$ and $d_{i}$ give the normalized contact distance $\left(\mathrm{d}_{\text {norm }}\right)$ [27]. The close contacts on the surface are marked by the red spots.

The shape of electron density surface around the molecular interactions is demonstrated by shape index. The weaker and longer bonds, excluding hydrogen bonds, are displayed as short range of dark and light color on the surface. The deep red color circles on the HS surface are defining the hydrogen bonding contacts. The blue regions on
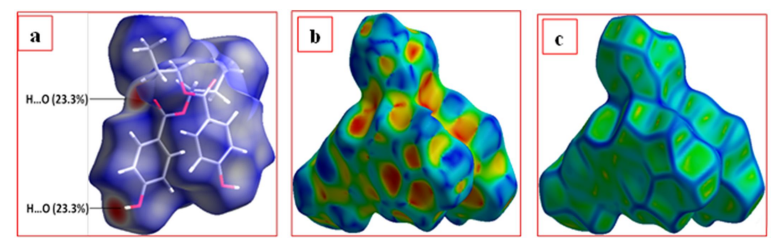

Fig. 6. Hirshfeld surface map of PHB : (a) $d_{\text {norm }}$, (b) shape index, (c) curvedness.

the HS show the longer contacts. The small range of area and light color on the surface represent a weaker and longer contact other than hydrogen bonds. The electron density surface curve around the molecule is pinpointed by the curvedness surface.

\subsection{Fingerprint}

The two-dimensional (2D) fingerprint plots of PHB elucidate the energetic clue for intermolecular interaction arrangement. The fingerprint plot is depicted in Fig. 7.

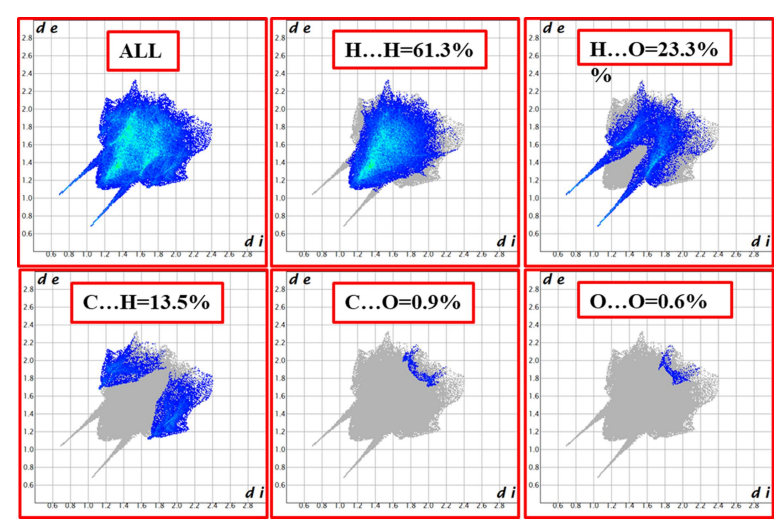

Fig. 7. Comparison of full fingerprint and decomposed fingerprint plots of PHB.

The decomposed fingerprint plot interactions give the interactions between two atoms and this has also been presented in Fig. 7. In the fingerprint plot, hydrogen bonding interactions $\mathrm{H}$...H $(61.3 \%)$ are very similar to other interactions. The H...H contact is essential for intermolecular contact. The H...H contacts in the fingerprint plot interactions are represented by a spike at the bottom. The O...H/H...O (23.3\%) interactions are pictured by a spike underlying at the plot. 
The fingerprint of a wing shape at the top right area represents the C...H (13.5\%) interactions. The small area in the right top represents the C...O $(0.9 \%)$ and O... O (0.6 \%) interactions, respectively. The sharp curved spike in the middle area illustrates the C...C (0.4\%) interactions.

\subsection{UV-Vis-NIR studies}

The optical transmittance spectrum of PHB recorded in the range of $200 \mathrm{~nm}$ to $1100 \mathrm{~nm}$ is shown in Fig. 8. The short wavelength cut-off occurs at $304 \mathrm{~nm}$. The transmittance is approximately $55 \%$ in the visible region. The optical transparency of organic single crystals of HA [28] p-MHB [29] and $\mathrm{DBCH}[30]$ is less than $50 \%$. The enhancement in the percentage of transmission may be attributed to a reduced scattering from structural and crystallographic defects [31]. This property makes the material highly usable for SHG applications [16].

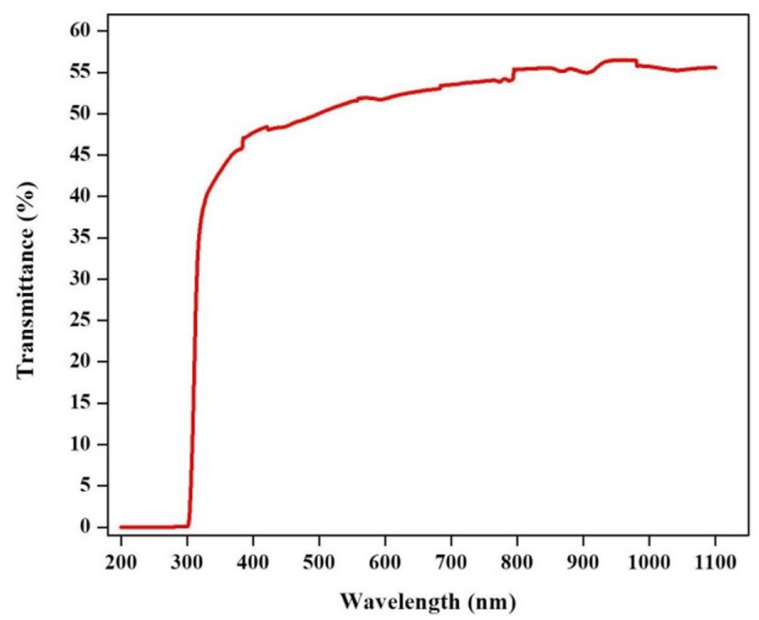

Fig. 8. UV-Vis-NIR spectrum of PHB.

\section{Computational details}

Density functional theory (DFT) is very useful for dealing with the electronic properties of the molecule. The studies on optimized geometries, electronic structures, dipole moments and polarizabilities for 2A5NPPS were carried out with Gaussian $09 \mathrm{~W}$ [32]. All the DFT calculations were performed by Becke three parameter and the LeeYang-Parr (B3LYP) [33] with 6-311++G(d,p) [34, 35] basis set. The optimized molecular structural parameters were used to calculate the other electronic properties. The electron density maps were made with the aid of Gauss View [36]. Natural bond orbital (NBO) analysis of the title compound was performed at B3LYP/6-311++G(d,p) level using the program [37] included in the Gaussian $09 \mathrm{~W}$ program package. Hirshfeld surface maps and fingerprint plots were generated from the crystallographic data using the Crystal Explorer 3.1 program package [38].

\section{Conclusions}

The PHB molecule has been theoretically analyzed for the first time in the literature. The optimized molecular structural parameters are well matched with the experimental data. The RMSD values show that the experimental and computed geometrical parameters are closely matched. The electronic density map of the PHB molecule gives the clue for intermolecular charge transfer within the molecule. The polarizabilty and hyperpolarizability of the PHB molecule have been calculated and the hyperpolarizability of PHB molecule is 8.9 times greater compared to the urea molecule. The Mulliken and NBO analysis shows the charge transfer within the PHB molecule. The Hirshfeld surface analysis shows the bond strength of the PHB molecule. From the results it has been concluded that the theoretical calculations help to find the new NLO materials.

\section{References}

[1] Zyss J., Chemla D.S., Nonlinear Optical Properties of Organic Molecules and Crystals, Academic Press, New York, 1987.

[2] Aruna S., Bhagavannarayana G., Palanisamy M., Thomas P.C., Varghese B., Sagayaraj P., J. Cryst. Growth, 300 (2007), 403.

[3] Dmitriev V.G., Gurzadyan G.G., Nikogosyan D.N., LoTSCH H.K.V., Handbook of Nonlinear Optical Crystals, Springer-Verlag, New York, 1999.

[4] Sun Z.H., Zhang L., Xu D., Wang X.Q., LiU X.J., Zhang G.H., Spectrochim. Acta A, 71 (2008), 663.

[5] Bosshard C., Spreiter R., Degiorgi L., Gunter P., Phys. Rev. B, 66 (2002), 205107.1 
[6] Shahab S., Filippovich L., Aharodnikova M., AlmodarResiYeH H.A., HaJiKolaee F.H., KUMAR R., MAShaYeKhi M., J. Mol. Struct., 1134 (2017), 530.

[7] Shahab S., Sheikhi M., Filippovich L., AnaTOL'Evich D.E., YAhyaei H., J. Mol. Struct., 1137 (2017), 335.

[8] Shahab S., Hajikolaee F.H., Filippovich L., Darroudi M., LoIKo V.A., Kumar R., BorzeHANDANi M.Y., Dyes Pigm., 129 (2016), 9.

[9] Shahab S., Almodarresiyeh H.A., Filippovich L., Hajikolaee F.H., Kumar R., Darroudi M., MAshaYeKhi M., J. Mol. Struct., 1119 (2016), 423.

[10] Sheikhi M., Shahab S., Filippovich L., YahyaeI H., Dikusar E., Khaleghian M., J. Mol. Struct., 1141 (2017), 703.

[11] Shahab S., Sheikhi M., Filippovich L., Kumar R., Dikusar E., Yahyaei H., Khaleghian M., $J$. Mol. Struct., 1148 (2017), 134.

[12] Wu K., Liu C., Mang C., Opt. Mater., 29 (2007), 1129.

[13] Kolev T.M., YancheVA D.Y., StamboliysKa B.A., Dimitrov M.D., Wortmann R., Chem. Phys., 348 (2008), 45.

[14] ViJayakumar T., Joe I.H., NaIR C.P.R., JayaKUMAR V.S., VAIDYAN V.K., JAYAKUMAR V.S., AIP Conference Proceedings, 2008.

[15] Zhou Y., MatsadiQ G., Wu Y., Xiao J., Cheng J., Acta Crystallogr. Section E, 66 (2010), o485.

[16] Karunagaran N., RAMASAmy P., RAMASAmY R.P., Bull. Mater. Sci., 37 (6) (2014), 1461.

[17] Solanki S.S.B., Perumal R.N., Suthan T., Materials Research Innovations, 2017, pp. 1-6.

[18] Thomson H.W., Torkington P., J. Chem. Soc., (1945) 640.

[19] Ravikumar C., Joe I.H., Jayakumar V.S., Chem. Phys. Lett., 460 (2008), 552.

[20] Fleming I., Frontier Orbitals and Organic Chemical Reactions, John Wiley and Sons, New York, 1976.

[21] Pearson R.G., Proc. Natl. Acad. Sci., 83 (1986), 8440.

[22] Tanak H., Agar A.A., BÜYÜKgüngÖR O., J. Mol. Struct., 1048 (2013), 41.

[23] Krishnakumar V., BalachandRan V., Chithambarathanu T., Spectrochim. Acta A, 62 (2005), 918.
[24] Nagabalasubramanian P.B., Periandy S., MoHAN S., Spectrochim. Acta A, 77 (2010), 150.

[25] Zhang R., Du B., Sun G., Sun Y.X., Spectrochim. Acta A, 75 (2010), 1115.

[26] Kleinman D.A., Phys. Rev., 126 (1977), 1962.

[27] Spackman M.A., JaYATIlaKa D., CrystEngComm, 11 (2009), 19.

[28] Kumar B.S., BabU K.R., Cryst. Res. Technol., 42 (2007), 607

[29] ViJayan N., BABU R.R., GunaseKaran M., GOPALAKRISHNAN R., RAMASAMY P., J. Cryst. Growth, 256 (2003), 174.

[30] Lakshmi K.U., Ramamurthi K., Cryst. Res. Technol., 40 (2005), 1165.

[31] Pandian M.S., Pattanaboonmee N., Ramasamy P., Manyum P., J. Cryst. Growth, 314 (2011), 207.

[32] Frisch M.J., TruCKS G.W., Schlegel H.B., Scuseria G.E., RobB M.A., Cheeseman J.R., Scalmani G., Barone V., Mennucci B., PetersSON G.A., Gaussian 09, Revision D. 01, Gaussian Inc., Wallingford CT, 2009.

[33] Becke A.D., J. Chem. Phys., 98 (1993), 5648.

[34] Petersion G.A., Bennett A., Tensfeldt T.G., Al-Laham M.A., Shirley W.A., Mantzaris J., $J$. Chem. Phys., 89 (1988), 2193.

[35] Petersson G.A., Al-Laham M.A., J. Chem. Phys., 94 (1991), 6081.

[36] Dennington R., Keith T., Millam J., GaussView, Version 5, Semichem Inc., Shawnee Mission KS, 2009.

[37] Glendening E.D., Badenhoop J.K., Reed A.E., CARPEnter J.E., Bohmann J.A., Morales C.M., WeINHOLD F., NBO 5.0, Theoretical Chemistry Institute, University of Wisconsin, Madison, 001.

[38] WolfF S.K., Grimwood D.J., McKinnon J.J., TURner M.J., JaYATILAKA D., SPACKMAN M.A., CrystalExplorer 3.1 (2013), University of Western Australia, Crawley, Western Australia, 2005 - 2013, http: //hirshfeldsurface.net/CrystalExplorer

Received 2017-12-14 Accepted 2019-04-23 